\title{
Machine learning with remote sensing data to locate uncontacted indigenous villages in Amazonia
}

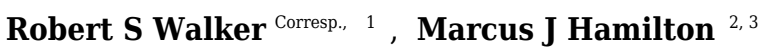 \\ ${ }^{1}$ Department of Anthropology, University of Missouri, Columbia, Missouri, USA \\ 2 Department of Anthropology, University of Texas at San Antonio, San Antonio, Texas, United States \\ 3 Santa Fe Institute, Santa Fe, New Mexico, USA \\ Corresponding Author: Robert S Walker \\ Email address: walkerro@missouri.edu
}

Background. The world's last uncontacted indigenous societies in Amazonia have only intermittent and often hostile interactions with the outside world. Knowledge of their locations is essential for urgent protection efforts, but their extreme isolation, small populations, and semi-nomadic lifestyles make this a challenging task.

Methods. Remote sensing technology with Landsat satellite sensors is a non-invasive methodology to track isolated indigenous populations through time. However, the small-scale nature of the deforestation signature left by uncontacted populations clearing villages and gardens has similarities to those made by contacted indigenous villages. Both contacted and uncontacted indigenous populations often live in proximity to one another making it difficult to distinguish the two in satellite imagery. Here we use machine learning techniques applied to remote sensing data with a training dataset of 500 contacted and 25 uncontacted villages.

Results. Uncontacted villages generally have smaller cleared areas, reside at higher elevations, and are farther from populated places and satellite-detected lights at night. A random forest algorithm with an optimally-tuned detection cutoff has a leave-one-out cross-validated sensitivity and specificity of over $98 \%$. A grid search around known uncontacted villages led us to identify 3 previously-unknown villages using predictions from the random forest model. Our efforts can improve policies toward isolated populations by providing better near real-time knowledge of their locations and movements in relation to encroaching loggers, settlers, and other external threats to their survival. 
1 Machine learning with remote sensing data to locate

2 uncontacted indigenous villages in Amazonia

3

4

5

6

7

\author{
Robert S. Walker ${ }^{1}$, Marcus J. Hamilton ${ }^{2,3}$ \\ ${ }^{1}$ Department of Anthropology, University of Missouri, Columbia MO \\ ${ }^{2}$ Department of Anthropology, University of Texas at San Antonio, San Antonio TX \\ ${ }^{3}$ Santa Fe Institute, Santa Fe, NM \\ Corresponding Author: \\ Robert S. Walker ${ }^{1}$
}

Department of Anthropology, University of Missouri, Columbia MO USA 65203

Email address: walkerro@missouri.edu

\title{
Abstract
}

Background. The world's last uncontacted indigenous societies in Amazonia have only intermittent and often hostile interactions with the outside world. Knowledge of their locations is essential for urgent protection efforts, but their extreme isolation, small populations, and seminomadic lifestyles make this a challenging task.

Methods. Remote sensing technology with Landsat satellite sensors is a non-invasive methodology to track isolated indigenous populations through time. However, the small-scale nature of the deforestation signature left by uncontacted populations clearing villages and gardens has similarities to those made by contacted indigenous villages. Both contacted and uncontacted indigenous populations often live in proximity to one another making it difficult to distinguish the two in satellite imagery. Here we use machine learning techniques applied to remote sensing data with a training dataset of 500 contacted and 25 uncontacted villages.

Results. Uncontacted villages generally have smaller cleared areas, reside at higher elevations, and are farther from populated places and satellite-detected lights at night. A random forest algorithm with an optimally-tuned detection cutoff has a leave-one-out cross-validated sensitivity and specificity of over $98 \%$. A grid search around known uncontacted villages led us to identify 3 previously-unknown villages using predictions from the random forest model. Our efforts can improve policies toward isolated populations by providing better near real-time knowledge of their locations and movements in relation to encroaching loggers, settlers, and other external threats to their survival. 


\section{Introduction}

41

42

43

44

45

46

47

48

49

50

51

52

53

54

55

56

57

58

59

60

61

62

63

64

65

66

67

68

69

70

71

72

73

74

75

76

77

78

79

The ongoing colonization of Amazonia has brought waves of epidemics and violence for centuries with severe consequences for indigenous populations (Bodard, 1974; Hemming, 1978; Hurtado et al. 2001; Hamilton, Walker \& Kesler, 2014). Amazingly, despite all the external pressures, remote areas in the upper Amazon watershed still support a number of remnant indigenous societies generally referred to as uncontacted or isolated populations (Vaz, 2001; Castillo, 2004; Ricardo \& Ricardo 2011). Despite these labels, intermittent and often hostile interactions with the outside world are commonplace (Wallace, 2011). Most governmental and non-governmental organizations promote no-contact policies for these isolated indigenous populations with the belief that they are safest if left to themselves (Walker \& Hill, 2015). However, encroachment from loggers, miners, settlers, and others is incessant and uncontacted societies represent the world's most critically endangered cultures (Walker, Kesler, \& Hill, 2016). There is a need for good information on their locations and movements in hopes of improving their survival prospects moving forward.

Our project is part of a longitudinal remote surveillance program to conduct scientific studies of indigenous demography and spatial ecology to facilitate informed decisions by policy makers that will increase protection efforts for isolated indigenous populations (Walker \& Hamilton, 2014; Walker, Hamilton \& Groth, 2014). Our central goal is to gather as much information on isolated indigenous populations as possible without attempting any direct contact (Kesler \& Walker, 2015). We maximize the use of available technologies to gather data remotely with no interference. Satellite imagery offers a safe, low-cost, and noninvasive method for studying population dynamics and spatial ecology of indigenous populations (Walker, Kesler, \& Hill, 2016). Similarly important is the need to understand spatial resource needs of indigenous societies in a region heavily impacted by deforestation, as well as the potential importance of connections among subpopulations, known to contribute to population viability (Levins, 1969; Hanski, 1999). The irreversible threats from large-scale habitat loss via deforestation and conversion of land to agriculture and pasture paint a bleak future for uncontacted populations (Fagan \& Shoobridge, 2005; Salisbury \& Fagan, 2013; Walker, Kesler \& Hill, 2016). The hope is that better data and methods can contribute improvements to this complex issue.

Applied machine learning is a vital tool for conservation work as a means to both collect and analyze more data at faster rates (Murray et al. 2018a). The growing use of machine learning methods to analyze large sets of biological, biophysical, spectral and climatological data has enabled accurate differentiation of the world's landscapes (Pettorelli et al. 2014). More germane to our work are forest classification projects (Hansen et al., 2013, Murray et al. 2018b). The Global Forest Change dataset was developed by classifying pixels using 15 or more highresolution global composite images as predictors, each developed from over 500,000 Landsat images (Hansen et al., 2013). 
80

81

82

83

84

85

86

87

88

89

90

91

92

93

94

95

96

97

98

99

100

101

102

103

104

105

106

107

108

109

110

111

112

113

114

115

116

117

118

119

The random forest algorithm is known to give excellent classification results and relatively quick processing speed (Du et al., 2015, Pal, 2005, Rodriguez-Galiano et al., 2012). Random forests (Breiman 2001) are an ensemble supervised learning method that builds multiple decisions trees used here for the classification of village class (uncontacted versus contacted). Random forests operate by constructing a multitude of decision trees. Some of the advantages of random forests are that they are robust to inclusion of features that are irrelevant to classification, and they are invariant to transformations of feature variables (Belgiu and Drăguţ 2016). For these reasons, the random forest algorithm is popular for remote sensing data given its accuracy, speed, and ability to handle high data dimensionality and multicollinearity.

\section{Materials \& Methods}

Data. We combined the exact locations (centroids) of 25 uncontacted and 500 contacted indigenous villages (Walker, Kesler \& Hill, 2016). More information about our general project along with high-resolution imagery for uncontacted villages is available at https://isolatedtribes.missouri.edu. The locations of uncontacted villages were originally derived from scouring high-resolution imagery using a combination of undergraduate helpers and various maps made by governmental and non-governmental agencies in Colombia, Ecuador, Peru and especially Brazil. Several additional locations have been pieced together from governmental reports and news stories stemming from overflights. Contacted villages are from the Brazilian government website (http://www.funai.gov.br/), and we included all of those that were in western Amazonia (west of 60 degrees longitude, Figure 1).

Hansen and colleagues' (2013) Global Forest Change (GFC) project provides small-scale deforestation at approximately $30 \mathrm{~m}$ resolution from Landsat sensors extending back to the year 2000. GFC version 1.5 goes up through the year 2017. We extracted the amount of detected deforestation in $2 \times 2 \mathrm{~km}$ squares surrounding each village's centroid and took the maximum area cleared in any one particular year from across the 17-year period. We refer to this measure as cleared area as it includes both the village and associated gardens but not those of neighboring villages. In addition, our dataset has other features, including regional population density in the nearest 100 square $\mathrm{km}$ (CIESIN, 2005), elevation at $30 \mathrm{~m}$ digital resolution from the Space Shuttle Radar Topography Mission (Rabus et al. 2003), and distance to populated places at $10 \mathrm{~m}$ resolution (Balk et al. 2006). We also included a local lights-at-night measure at $3 \mathrm{~km}$ resolution (Pritchard, 2017, from https://earthobservatory.nasa.gov) using the distance from village centroid to the nearest detected lights. Finally, distance to rivers of all the different Strahler stream orders using the Global Self-consistent, Hierarchical, High-resolution Geography Database (Wessel \& Smith, 1996), along with the minimum distance to combined rivers of Strahler stream orders 1 , 2 , and 3 , giving a total of 11 features used to train algorithms. 
121

122

123

124

125

126

127

128

129

130

131

132

133

134

135

136

137

138

139

140

141

142

143

144

145

146

147

148

149

150

151

152

153

154

155

156

157

158

159

Models. Machine learning algorithms were performed with the R package caret. We found that an untuned random forest algorithm had a fairly high combination of sensitivity (true positive rate) and specificity (true negative rate) in the 0.8 to 0.9 range. As mentioned above, random forest algorithm is an ensemble classifier that produces multiple decision trees, using a randomly selected subset of training samples and variables. Other algorithms such as neural networks, extreme gradient boosting tree, and lasso logistic regression were also relatively-high performing but gave slightly lower values on one or the other metric.

The target classes in our sample are imbalanced with only $4.8 \%$ of villages in the sample being uncontacted. During model training we noticed that varying the detection cutoff (also known as the threshold) that classifies villages into one class or the other had large effects on the results (the default cutoff is 0.5 majority rule). In addition, common loss metrics such as the area under the ROC curve or the F1 score tended to give either high specificity or sensitivity with our data, but not both.

To address the imbalanced data issue and improve model performance, we used a random forest algorithm that iteratively tuned the cutoff value such as to simultaneously maximize both specificity (true negative rate) and sensitivity (true positive rate). In other words, we instituted cost-sensitive learning into the random forest (Elkan, 2001; Zadrozny et al. 2003; Khoshgoftaar et al. 2007). The loss metric we used for training is the distance from a perfect model of sensitivity of 1 and specificity of 1 . We used 1,000 trees with 2 variables available for splitting at each tree node. To evaluate models we used a leave-one-out cross-validation (non-nested) looped over a range of cutoffs from 0.01 to 0.99 in increments of 0.01 . Raising the cutoff value means a higher level of evidence (i.e., more decision trees out of the total 1,000 trees that comprise the random forest) is needed to assign the positive class (uncontacted) so it decreases sensitivity and increases specificity. Here a sensitive cutoff of 0.2 yields a minimal distance metric and the desired combination of high sensitivity and specificity metrics (Figure 2).

\section{Results}

Our random forest algorithm, with an optimally-tuned cutoff of 0.2 , yields a sensitivity of 1.0 and a specificity of 0.98 using leave-one-out cross-validation. This means that all uncontacted villages are correctly classified and $98 \%$ of the contacted villages are correctly classified. Therefore, our model has a strong ability to automatically distinguish between contacted and uncontacted villages. In order of descending variable importance, uncontacted villages have 1) smaller cleared areas, 2) longer distances from lights, 3) higher elevation, 4) longer distances to populated places, 5) lower regional population density, 6) longer distances from rivers of all Strahler stream orders up to and including 3, and 7) shorter distances to rivers of levels 4 and 5. Figure 3 shows density plot comparisons for the top 4 features in terms of variable importance. 
160

161

162

163

164

165

166

167

168

169

170

171

172

173

174

175

176

177

178

179

180

181

182

183

184

185

186

187

188

189

190

191

192

193

194

195

196

197

198

199

Given the success of our algorithm during cross-validation, we then moved to implement it for predictive purposes. We did a grid search of all $2 \times 2 \mathrm{~km}$ squares within a $100 \mathrm{~km}$ radius of the 5 clusters of known uncontacted villages (Figure 1). This approach does produce a high number of false positives created by natural clearings (e.g., landslides, windfalls, etc.). Fortunately, most natural clearings can be eliminated by simply removing all clearings that are less than 0.5 ha. This left us with a sample of 20 clearings. Of these we were able to obtain high resolution imagery for 8 of these and 3 contained newly-identified villages. One of these in Colombia appears to be currently inhabited given that it has a single longhouse structure and shows recently made clearings in Global Land Analysis and Discovery (GLAD, Tyukavina et al. 2016). The GLAD alert system processes Landsat imagery as it becomes available to identify tree cover change in near real-time. This is an invaluable system for monitoring both recent activity by uncontacted villages, as well as encroaching deforestation from outsiders.

The other two newly-discovered sites are historical villages. One is from the uncontacted Yanomami in northern Brazil inhabited from around year 2000 or earlier and until 2004. The other is from Pano speakers on the border between Peru and Brazil and was probably inhabited during a similar time period. The other 5 possible locations identified by the random forest predictions with high resolution imagery available all appeared to be natural. Therefore, we estimate our testing precision with this small sample as 0.375 (3 true positives divided by 8 total cases).

\section{Discussion}

We used deforestation data from Landsat satellites to train algorithms to identify the locations of uncontacted indigenous groups in Amazonia as part of an ongoing effort to better understand their conservation status and threats. Our results show that uncontacted villages have smaller cleared areas, reside at higher elevations, and are farther from populated places and satellitedetected lights at night. Our random forest algorithm with an optimally-tuned cutoff has crossvalidated performance metrics of over $98 \%$.

The case of the uncontacted Yanomami (also known as the Moxihatetea) is a good example of the importance of a near real-time monitoring system. Their previous village was abandoned in late 2014 and the Brazilian indigenous agency (FUNAI) and the Yanomami indigenous association (Hutukara) were particularly worried that some disaster had befallen them since much of the nearby area has seen invasions by gold miners. For a year and a half their whereabouts were unknown. We began looking for them using Landsat data, but it was the remote sensing fire alerts (FIRMS, Davies et al. 2009) that first alerted us to their exact location. We tasked a DigitalGlobe satellite image on May 12, 2016 and were relieved to find out that they were alive and well and clearing large gardens. The number of sections in their shabono village 
200

201

202

203

204

205

206

207

208

209

210

211

212

213

214

215

216

217

218

219

220

221

222

223

224

225

226

227

228

229

230

231

232

233

234

235

236

237

238

239

structure had increased from 16 to 17 . We relayed this information on to FUNAI and Hutukara who then organized a flyover to officially confirm the location.

Remote sensing provides many advantages over flyovers, and we actually do not recommend them. As we have shown, the information provided solely by remote sensing is sufficient to identify uncontacted villages. Remote sensing is safe, low-cost, and noninvasive, while flyovers are not. Population estimates are also crucial information for assessing trends in the demographic health of isolated populations by measuring areas of fields, villages, and houses in satellite imagery. Heads-up digitization of satellite imagery provides better population estimates than do flyovers where most people are not visible because many hide or run away in fear. Remote sensing offers the benefits of time-stamped evidence of occupation of areas inhabited by isolated populations, along with movements through time (Walker, Kesler \& Hill, 2016).

\section{Conclusions}

A dozen easily obtainable remote sensing measures allowed our random forest algorithm to successfully classify uncontacted versus contacted villages. Extending the algorithm to make predictions in a grid search greatly accelerates our ability to find and identify the locations of uncontacted villages. Moving forward we anticipate using an even lower cutoff value because the decreasing costs in satellite imagery make false positives from a more sensitive algorithm relatively cheap to evaluate and discard. We anticipate that this method will become the primary means by which to track and locate these same uncontacted villages, as well as undiscovered locations of uncontacted villages.

One shortcoming of our classification model when applied to searching through unlabeled satellite imagery is that it was not designed to classify natural landslides, windfalls, or riverbank clearings. All of these natural processes also create deforestation signatures that further complicate our searches. Future work could well include these, but in the meantime we filter our predictions based on cleared area because natural clearings tend to be less than 0.5 ha while most uncontacted villages have larger areas than that.

Our research is vital and timely as isolated groups are among the last remaining small-scale subsistence populations living in a traditional lifestyle. The enormous and mounting pressure from external threats create the possibility that isolated populations will disappear in the near future. Better monitoring and tracking with remote sensing are tools that might provide more informed conservation decisions concerning increased protection and land rights for the world's most critically-endangered human cultures.

\section{Acknowledgements}


240 We thank Mark Flinn and the Comparative Methods course at the University of Missouri for 241 their help and suggestions.

242

243

244

245

246

247

248

249

250

251

252

253

254

255

256

257

258

259

260

261

262

263

264

265

266

267

268

269

270

271

272

273

274

275

276

277

278

279

280

281

282

283

\section{References}

Balk DL, Deichmann U, Yetman G, Pozzi F, Hay SI, Nelson A. 2006. Determining global

population distribution: methods, applications and data. Advances in Parasitology 62:119-156.

Belgiu M, Drăguţ L. 2016. Random forest in remote sensing: A review of applications and future directions. ISPRS Journal of Photogrammetry and Remote Sensing 114:24-31.

Bodard L. 1974. Green hell: massacre of the Brazilian Indians. New York: Dutton.

Breiman L. 2001. Random forests. Machine Learning 45:5-32.

Castillo BH. 2004. Indigenous peoples in isolation in the Peruvian Amazon. Copenhagen: International Work Group for Indigenous Affairs.

Center for International Earth Science Information Network (CIESIN). 2005. Gridded population of the world: population density grid. Columbia University, Centro Internacional de Agricultura Tropical.

Davies DK, Ilavajhala S, Wong MM, Justice CO. 2009. Fire information for resource management system: archiving and distributing MODIS active fire data. IEEE Transactions on Geoscience and Remote Sensing 47:72-79.

Du P, Samat A, Waske B, Liu S, Li Z. 2015. Random forest and rotation forest for fully polarized SAR image classification using polarimetric and spatial features. ISPRS Journal of Photogrammetry and Remote Sensing 105:38-53.

Elkan C. 2001. The foundations of cost-sensitive learning. Proceedings of the IEEE International Joint Conference on Artificial Intelligence 17:973-978.

Fagan C, Shoobridge D. 2005. An investigation of illegal mahogany logging in Peru's Alto Purús National Park and its surroundings. Durham NC: ParksWatch.

Hamilton MJ, Walker RS, Kesler D. 2014. Crash and rebound of indigenous populations in lowland South America. Sci. Rep. 4, 4541.

Hanski I. 1999. Metapopulation ecology. Oxford: Oxford University Press.

Hansen MC, Potapov PV, Moore R, Hancher M, Turubanova SA, Tyukavina A, Thau D, Stehman SV, Goetz SJ, Loveland TR, Kommareddy A. 2013. High-resolution global maps of 21st-century forest cover change. Science 342:850-853. 
284

285

286

287

288

289

290

291

292

293

294

295

296

297

298

299

300

301

302

303

304

305

306

307

308

309

310

311

312

313

314

315

316

317

318

319

320

321

322

323

324

325

326

327 328

Hemming J. 1978. Red gold: the conquest of the Brazilian Indians. Cambridge: Harvard University Press.

Hurtado AM, Hill KR, Kaplan H, Lancaster J. 2001. The epidemiology of infectious diseases among South American Indians: a call for guidelines for ethical research. Curr. Anth. 42:425432.

Kesler DC, RS Walker. 2015. Geographic distribution of isolated indigenous societies in Amazonia and the efficacy of indigenous territories. PLOS ONE 10:e125113.

Khoshgoftaar TM, Golawala M, Van Hulse J. 2007. An empirical study of learning from imbalanced data using random forest. IEEE Artificial Intelligence Tools 2:310-317.

Levins, R. 1969. Some demographic and genetic consequences of environmental heterogeneity for biological control. Bull. Entomol. Soc. Am. 15:237-240.

Murray NJ, Keith DA, Bland LM, Ferrari R, Lyons MB, Lucas R, Pettorelli N, Nicholson E. 2018a. The role of satellite remote sensing in structured ecosystem risk assessments. Science of the Total Environment 619:249-257.

Murray NJ, Keith DA, Simpson D, Wilshire JH, Lucas RM. 2018b. REMAP: An online remote sensing application for land cover classification and monitoring. Methods in Ecology and Evolution. 9:2019-2027.

Pal M. 2005. Random forest classifier for remote sensing classification. International Journal of Remote Sensing 26:217-222.

Pappalardo SE, De Marchi M, Ferrarese F. 2013. Uncontacted Waorani in the Yasuní Biosphere Reserve: geographical validation of the Zona Intangible Tagaeri Taromenane (ZITT). PLoS ONE 8:e66293.

Pettorelli N, Laurance WF, O'Brien TG, Wegmann M, Nagendra H, Turner W. 2014. Satellite remote sensing for applied ecologists: opportunities and challenges. Journal of Applied Ecology $51: 839-848$.

Pritchard SB. 2017. The trouble with darkness: NASA's Suomi satellite images of earth at night. Environmental History 22:312-330.

Rabus B, Eineder M, Roth A, Bamler R. 2003. The shuttle radar topography mission - a new class of digital elevation models acquired by spaceborne radar. ISPRS Journal of Photogrammetry and Remote Sensing 57:241-262.

Ricardo B, Ricardo F. 2011. Povos indígenas no Brasil. São Paulo: Instituto Socioambiental. 
329 Rodriguez-Galiano VF, Ghimire B, Rogan J, Chica-Olmo M., Rigol-Sanchez JP. 2012. An 330 assessment of the effectiveness of a random forest classifier for land-cover classification. ISPRS

331

332

333

334

335

336

337

338

339

340

341

342

343

344

345

346

347

348

349

350

351

352

353

354

355

356

357

358

359

360

361
Journal of Photogrammetry and Remote Sensing 67:93-104.

Salisbury DS, Fagan C. 2013. Coca and conservation: cultivation, eradication, and trafficking in the Amazon borderlands. GeoJ. 78:41-60.

Tyukavina A, Hansen MC, Potapov PV, Krylov AM, Goetz SJ. 2016. Pan-tropical hinterland forests: mapping minimally disturbed forests. Global Ecology and Biogeography 25:151-163.

Vaz A. 2011. Isolados no Brasil. Política de estado: da tutela para a política de direitos - uma questão resolvida? Brasília: Estação Gráfica.

Walker RS, DC Kesler, KR Hill. 2016. Are isolated indigenous populations headed toward extinction? PLoS ONE 11:e0150987.

Walker RS, Hill KR. 2015. Protecting isolated tribes. Science 348:1061.

Walker RS, Hamilton MJ. 2014 Amazonian societies on the brink of extinction. Am. J. Hum. Bio. 26:570-572.

Walker RS, Hamilton MJ, Groth AA. 2014. Remote sensing and conservation of isolated indigenous villages in Amazonia. Royal Society Open Science 1:140246.

Wallace S. 2011. The Unconquered: In Search of the Amazon's Last Uncontacted Tribes. New York: Random House LLC.

Wessel P, Smith WHF. 1996. A global, self-consistent, hierarchical, high-resolution shoreline database. J. Geophys. Res. 101:8741-8743, doi:10.1029/96JB00104.

Zadrozny B, Langford J, Abe N. 2003. Cost-sensitive learning by cost-proportionate example weighting. Proceedings of the IEEE International Conference on Data Mining 3:435-442. 
Figure 1 (on next page)

Figure 1. Map of study locations.

Map of 500 contacted indigenous villages in Brazil and 25 uncontacted indigenous villages in Brazil, Colombia, Ecuador, and Peru that were included in the study. 


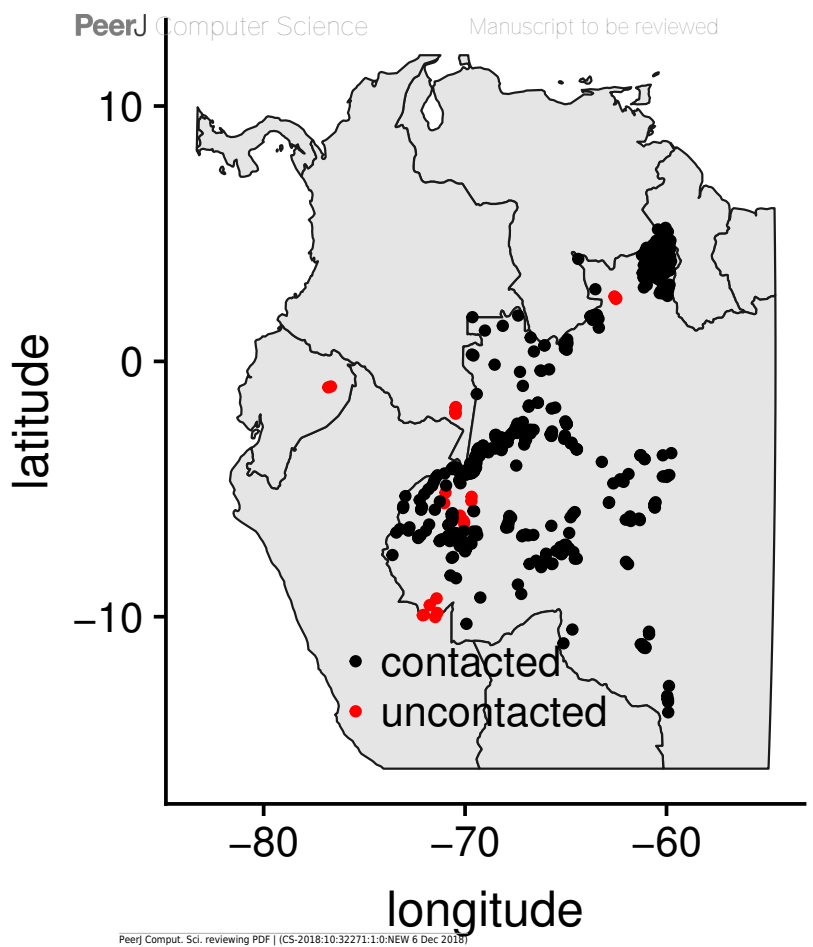




\section{Figure 2 (on next page)}

Model metrics obtained from training the random forest model across a range of cutoffs from 0.01 to 0.99 in increments of 0.01 .

To train the random forest model we used leave-out-out cross-validation across a range of cutoffs from 0.01 to 0.99 in increments of 0.01 . Raising the cutoff value means a higher level of evidence is needed to assign the positive class (uncontacted), which decreases sensitivity (true positive rate) and increases specificity (true negative rate). Here the optimal cutoff (0.2) gives a perfect cross-validated sensitivity of 1.0 and a specificity of 0.98 . The distance is the distance from a perfect model which is minimized during training. 


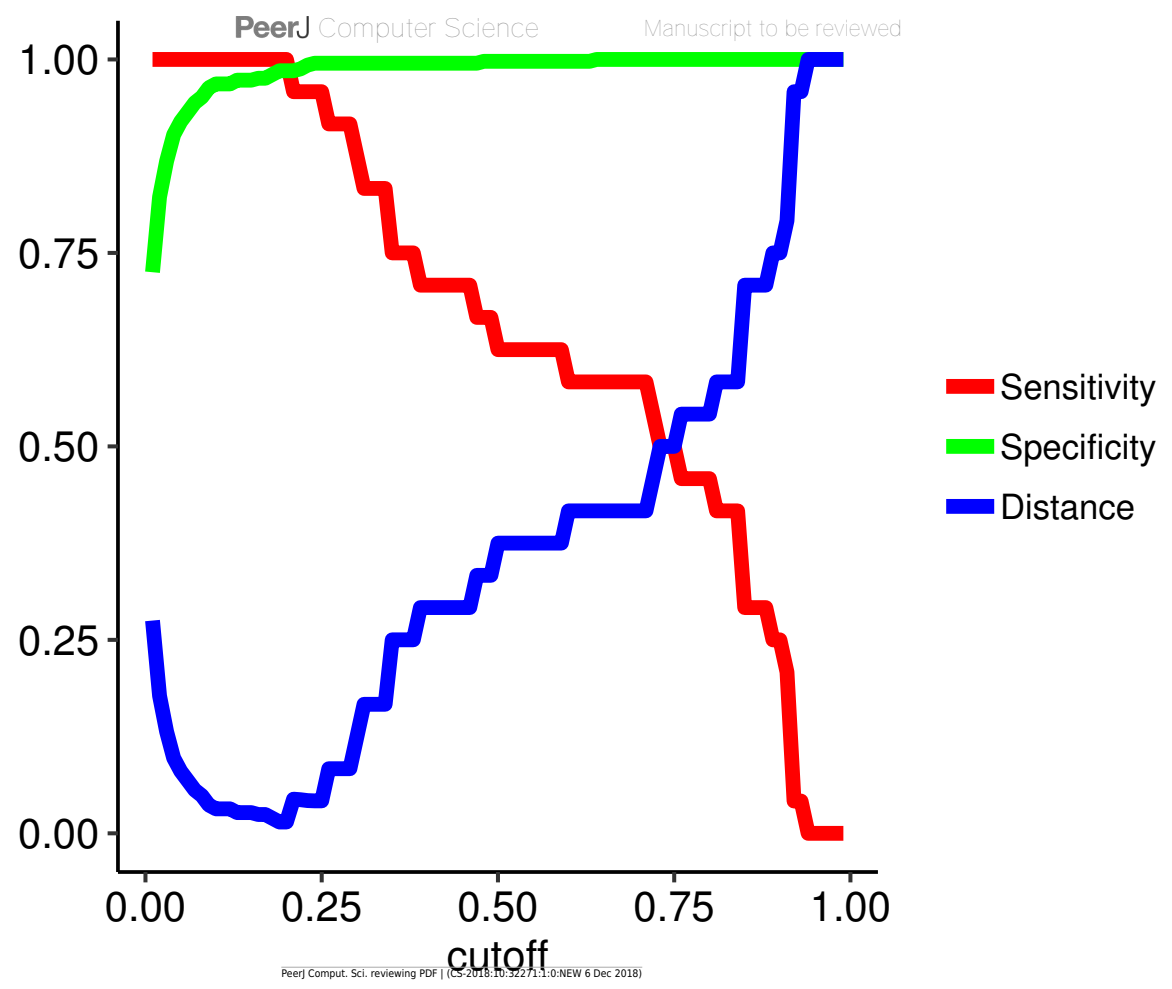


Figure 3 (on next page)

Smoothed kernel density plots comparing uncontacted to contacted indigenous villages.

The top 4 distinguishing features in terms of variable importance in the random forest model are uncontacted vilages have (A) smaller cleared areas, (B) farther distances to satellitedetected lights at night, (C) higher elevation, and (D) farther distances to populated places, on average. Plots $A$ and $C$ are best visualized on log scales. 
PeerJ Compute Scienacied $\square$ uncobltactedcript to be reviewed
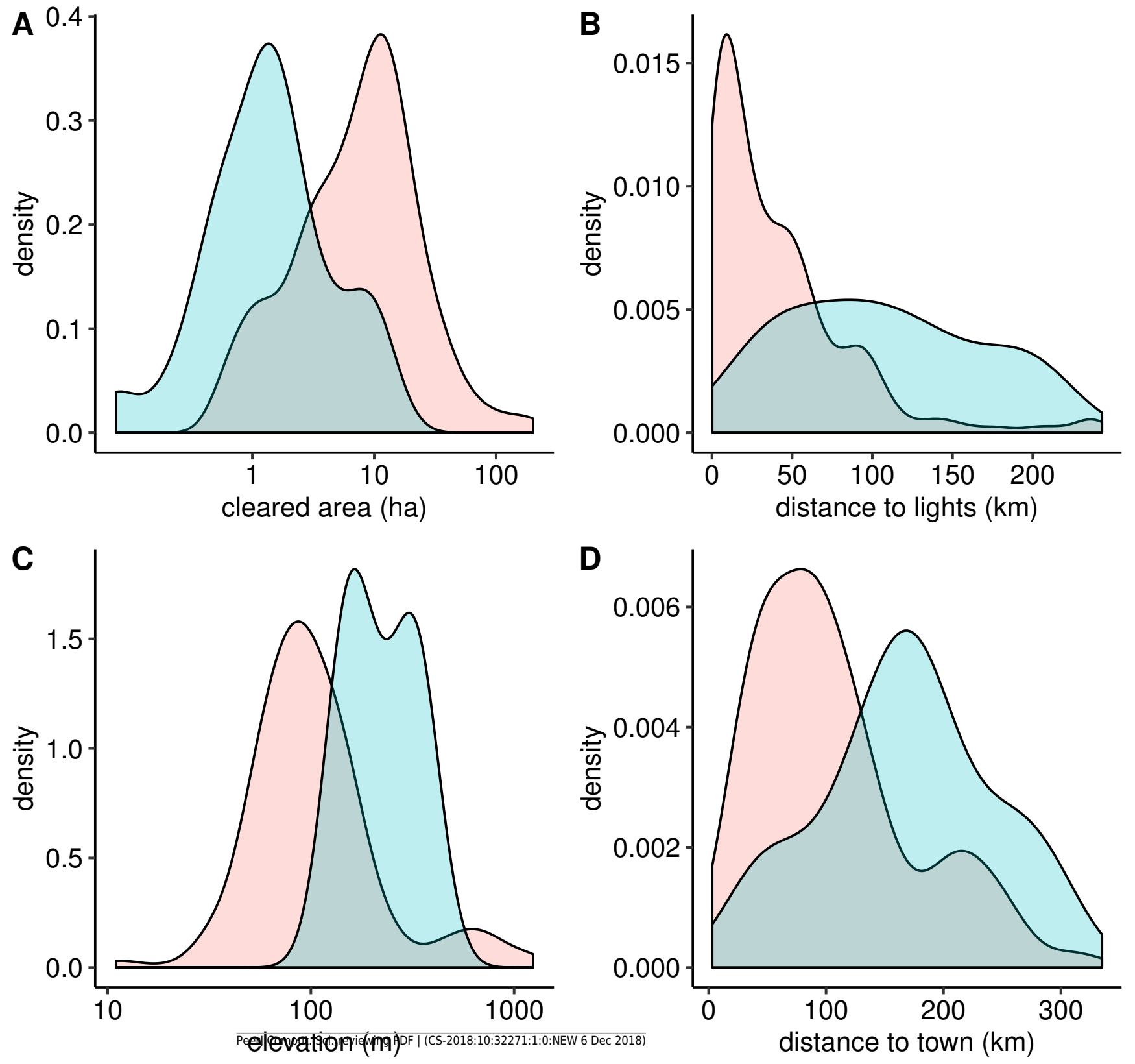